\title{
Unprecedented C-Methylation at the 2-Position of 2-Carboxy- 4-Chromanones - A Case Study with the Corey-Chaykovsky Reagent
}

\author{
Subrata Ghosh \\ Nellore Bhanu Chandar \\ Debayan Sarkar \\ Manoj Kumar Ghosh \\ Bishwajit Ganguly* \\ Indrajit Chakraborty* \\ Synlett 2014, 25, 2649
}

The authors acknowledge Prof. R. V. Venkateswaran, IACS, Jadavpur, Kolkata, for his facility support. 